\title{
Forward jets and saturation effects within high energy factorization
}

\section{Piotr KOTKO*†}

The H. Niewodniczański Institute of Nuclear Physics

Polish Academy of Sciences

Radzikowskiego 152, 31-342 Cracow, Poland

E-mail: piotr.kotko@ifj.edu.pl

\begin{abstract}
We present results for forward jet production in $\mathrm{p}-\mathrm{p}$ and $\mathrm{p}-\mathrm{Pb}$ collisions at LHC within the High Energy Factorization (HEF) framework, obtained using new Monte Carlo programs. The HEF framework incorporates a convolution of unintegrated gluon densities and off-shell matrix elements. We briefly discuss both components: the relevant evolution equations and methods to obtain tree level off-shell matrix elements in a gauge invariant way. In particular, a new Wilson line-based method for off-shell multi-leg amplitudes is presented. The numerical results for forward-forward dijets and forward-central trijets are discussed.
\end{abstract}

XXII. International Workshop on Deep-Inelastic Scattering and Related Subjects 28 April - 2 May 2014

Warsaw, Poland

\footnotetext{
*Speaker.

${ }^{\dagger}$ In collaboration with A. van Hameren, K. Kutak, C. Marquet, S. Sapeta.
} 


\section{Introduction}

It is known that hadronic collisions with accompanying production of jets in the forward rapidity regions are potentially ideal tools for looking for so-called small $x$ effects. By these we simply mean a necessity of a systematic resummation of $\alpha_{s} \log (1 / x)$ perturbative terms, where $x$ is a fraction of the initial state hadron momentum carried by the parton participating in the "hard collision" and is numerically small. The simplest form of such resummation leads to Balitsky-FadinKuraev-Lipatov (BFKL) evolution equation [1-3], but there are various improvements aiming either to resolve certain drawbacks of the BFKL approach, or to match BFKL with the well-tested Dokshitzer-Gribov-Lipatov-Altarelli-Parisi (DGLAP) evolution equation which works for larger $x$. The former group includes the Balitsky-Kovchegov (BK) equation $[4,5]$ (or its improved version incorporating running strong coupling, $\operatorname{rcBK}[6,7]$ ) which accounts for the gluon saturation phenomenon [8]. For the latter group we can include Ciafaloni-Catani-Fiorani-Marchesini (CCFM) equation [9-11]. There is also a more general approach extending the CCFM to a nonlinear equation [12], or slightly less complicated equation supplementing the momentum space BK equation with certain DGLAP terms and so-called kinematic constraint [13, 14]. The latter equation was used in [15] to obtain an unintegrated gluon density (UGD), which in what follows will be called KS UGD. Primarily due to the kinematic constraint it has a capability to deal with large $p_{T}$ jets, remaining in the same time a small- $x$ approach.

A theoretic construct that makes a practical use of UGDs is so-called $k_{T}$ or high energy factorization (HEF), which convolutes transverse momentum dependent UGDs with hard sub-processes defined with initial states being off-shell. The HEF notion is quite general and actually may correspond to various factorization formulae; for instance for inclusive dijet production [8] or for heavy quark pair production [16, 17]. These approaches are not all-orders QCD theorems, and for hadron-hadron collisions they are expected to be violated (see e.g. [18-22]). Nevertheless, they are proven to be very useful in an actual phenomenology, with the restriction that UGDs might not be universal. For the forward jet processes the following HEF factorization formula can be used

$$
d \sigma_{A B \rightarrow X}=\int \frac{d^{2} k_{T A}}{\pi} \int \frac{d x_{A}}{x_{A}} \int d x_{B} \sum_{b} \mathscr{F}_{g^{*} / A}\left(x_{A}, k_{T A}, \mu\right) f_{b / B}\left(x_{B}, \mu\right) d \hat{\sigma}_{g^{*} b \rightarrow X}\left(x_{A}, x_{B}, k_{T A}\right),
$$

where $\mathscr{F}_{g^{*} / A}$ is the UGD, $f_{b / B}$ are the collinear parton distribution functions and $b$ runs over gluon and all the quarks that can contribute to the production of multiparticle state $X$ (see also [23]). The off-shell gauge invariant matrix elements for multiple final states (residing in $d \hat{\sigma}_{g^{*} b \rightarrow X}$ ) will be discussed in Sec. 2. It is implicitly assumed that $x_{B} \gg x_{A}$ in order to populate the forward rapidity region. Often, the UGDs do not depend on the hard scale $\mu$, however this dependence is important for certain observables.

\section{Off-shell gauge invariant amplitudes}

When the final states $X$ in (1.1) involve gluons, the off-shell tree-level amplitude calculated from the standard Feynman diagrams is in general not gauge invariant. There are a few methods to overcome this: the Lipatov's effective action and the resulting Feynman rules [24], the methods suitable for automatic numeric calculation [25-27] and the method of [28] using matrix elements of Wilson lines. Below we shall briefly describe the last method. 
The basic idea is to find the gauge invariant amplitude $\tilde{\mathscr{M}}_{e_{1} \ldots e_{n}}\left(k_{1}, \ldots, k_{n} ; X\right)$ for the process $g^{*}\left(k_{1}, e_{1}\right) \ldots g^{*}\left(k_{n}, e_{n}\right) \rightarrow X$ which becomes the standard QCD amplitude $\mathscr{M}_{e_{1} \ldots e_{n}}\left(k_{1}, \ldots, k_{n} ; X\right)$ if the gluons are taken on-shell. Here, $k_{i}$ and $e_{i}$ are momenta and "polarization" vectors of off-shell gluons (mutually transverse in the Feynman gauge). The amplitude $\tilde{\mathscr{M}}$ can be found as [28]

$$
\begin{aligned}
\left\langle 0\left|\mathfrak{R}_{e_{1}}^{c_{1}}\left(k_{1}\right) \ldots \mathfrak{R}_{e_{n}}^{c_{n}}\left(k_{n}\right)\right| X\right\rangle & =\delta\left(k_{1} \cdot e_{1}\right) \ldots \delta\left(k_{n} \cdot e_{n}\right) \\
& \delta^{4}\left(k_{1}+\ldots+k_{n}-X\right) \tilde{\mathscr{M}}_{e_{1} \ldots e_{n}}\left(k_{1}, \ldots, k_{n} ; X\right)+\text { disconnected diagrams },
\end{aligned}
$$

where the Wilson line operators (one for each off-shell gluon) are defined as

$$
\mathfrak{R}_{e_{i}}^{c_{i}}\left(k_{i}\right)=\int d^{4} y e^{i y \cdot k_{i}} \operatorname{Tr}\left\{\frac{1}{\pi g} t^{c_{i}} \mathscr{P} \exp \left[i g \int_{-\infty}^{\infty} d s \frac{d z_{i \mu}(s)}{d s} A_{b}^{\mu}(z) t^{b}\right]\right\}
$$

with $t^{a}$ being the color generators. In order to keep the integrals over $d s$ finite and derive formally the generalized functions appearing in (2.1) the path $z_{i}(s)$ in (2.2) is parametrized as

$$
z_{i}^{\mu}(s)=y^{\mu}+\frac{2}{\varepsilon} \tanh \left(\frac{\varepsilon s}{2}\right) e_{i}^{\mu}, \quad s \in(-\infty, \infty) .
$$

The path becomes infinite and straight in the $\varepsilon \rightarrow 0$ limit leading to the transverse delta functions $\delta\left(e_{i} \cdot k_{i}\right)$ and the eikonal propagators of the form $i /\left(e_{i} \cdot p+i 0^{+}\right)$.

The tree-level matrix elements (2.1) can be calculated automatically using OGIME program [29]. The results coincide with the method of [27] suitable for fast numeric computations. Moreover, for a certain choice of "polarization" vectors $e_{i}$ we recover the Lipatov's vertices for reggeons scatterings.

\section{Numerical results for forward jets in $\mathbf{p}-\mathrm{p}$ and $\mathrm{p}-\mathrm{Pb}$ collisions}

\subsection{Forward-forward dijets}

In order to access the saturation regime we propose to look at dijet events, where two reconstructed jets lie in the forward rapidity region $3.2<\left|y_{i}\right|<4.9, i=1,2$ [30]. The results were obtained using three independent Monte Carlo programs: forward [31], a program implementing the method of [26] and LxJet [32]. The potential collinear singularities were killed by choosing anti- $k_{T}$ algorithm with $R=0.5$ (identical here to a proper cut on the $\phi-\eta$ plane). In order to analyse the sensitivity of our results to the particular model for UGD we have used KS and rcBK evolution equations. We present the results in Fig. 1 We see a strong suppression of the nuclear modification factors (i.e. the ratios of $\mathrm{p}-\mathrm{p}$ to $\mathrm{p}-\mathrm{Pb}$ cross sections) indicating the saturation effects. In particular, the $p_{T}$ spectrum of the soft jet is especially sensitive: it is suppressed over the whole kinematic region.

\subsection{Forward-central dijets and trijets as a tool to access large gluon off-shellness}

The saturation phenomenon is related to rather low $k_{T}$ region of the UGD. It is, however, also very interesting to look at larger $k_{T}$, i.e. at the larger gluon off-shellnes. An examplary observable is three jet production, where the softest jet is in the forward region $3.2<y_{3}<4.7$, while the remaining two jets are in the central region $\left|y_{1,2}\right|<2.8$ and balance each other within 

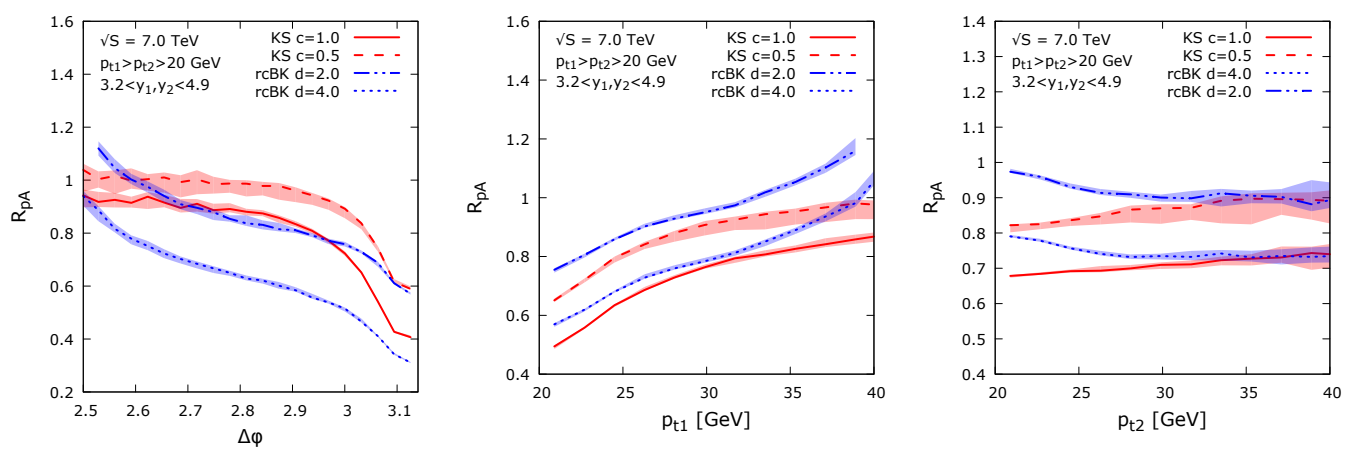

Figure 1: The results for nuclear modification ratios for forward-forward dijet production within HEF framework using KS and rcBK UGDs. The uncertainty bands come from the scale choice uncertainty. The parameters $d$ and $c$ influence the strength of the nonlinear term in the evolution equation for the lead. We refer to [30] for further details.
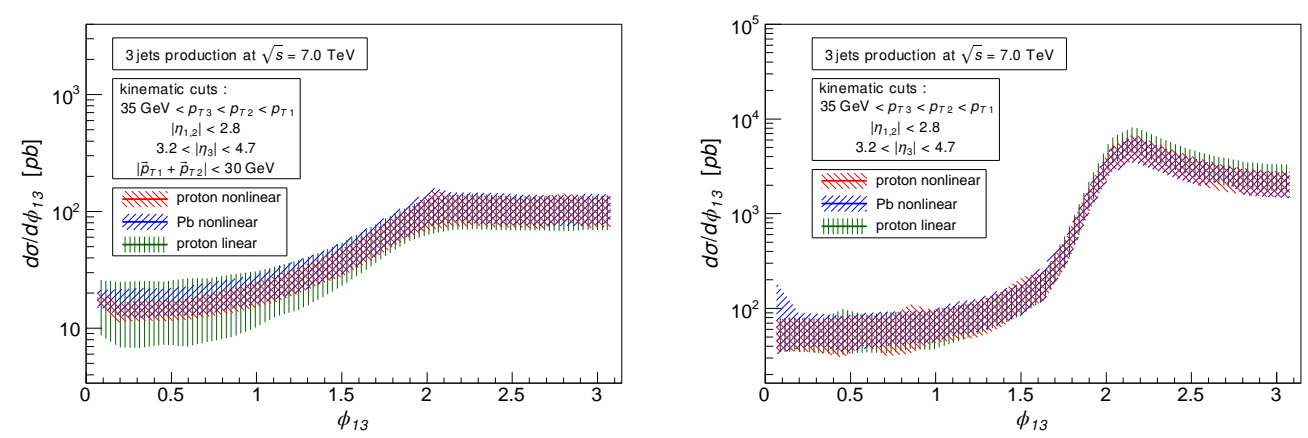

Figure 2: Azimuthal decorrelations for forward-central three jet production. The two hardest jets are in the central detector, while the softest jet is in the forward region. For the left plot an additional cut is applied on the central jets, namely we require that they should almost balance each other. This flattens the distribution (left) comparing to the case without this cut (right). For more details refer to [33].

a certain cut, $\left|\vec{p}_{T 1}+\vec{p}_{T 2}\right|<D_{\text {cut }}$ [33]. Then, the forward jet accesses almost directly the large $k_{T}$ distribution (here $k_{T}>35 \mathrm{GeV}$ ) of small- $x$ UGD. The azimuthal decorrelations in that configuration are presented in Fig. 2.

In the future it could be possible to access even smaller $x$ by using a possible upgrade of the CMS CASTOR detector to be able to reconstruct the jets. Then, for instance, one could consider forward dijets when the softest jet is in the CASTOR while the harder (but still forward) in HF. The cross section calculations are presented in Fig. 3.

In the end, let us comment, that for larger $k_{T}$ certain observables, in particular the azimuthal decorrelations, are sensitive to an additional kind of resummation of the Sudakov type. This is directly linked to the hard scale dependence of the UGD. Such a study has been done recently in [34], where the calculation for decorrelations of forward-central dijets were calculated in HEF supplemented in this kind of resummation and compared with the recent CMS data [35]. We refer to the talk of K. Kutak for more details. 

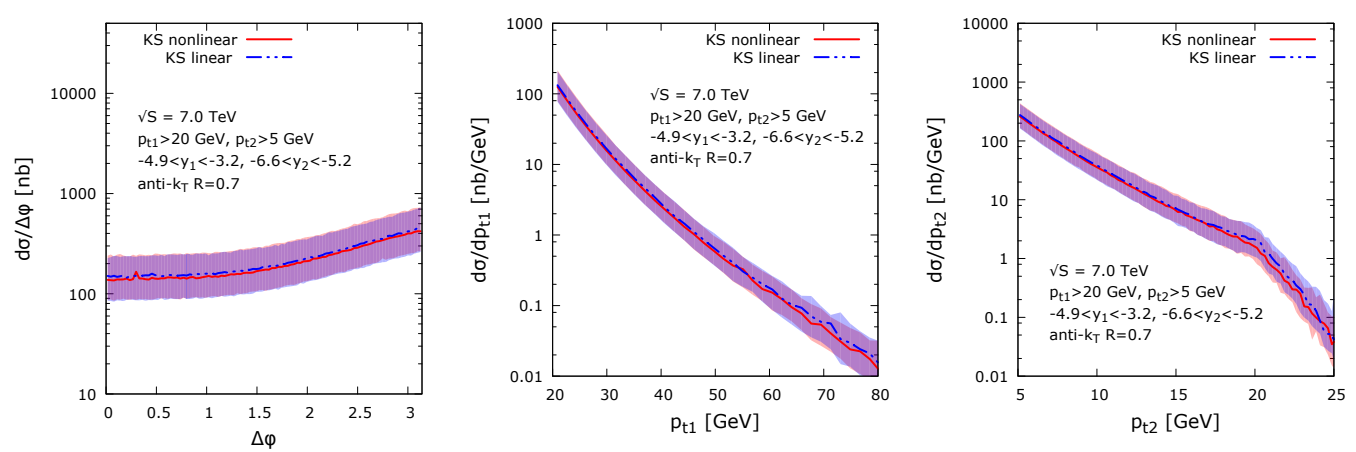

Figure 3: The result for dijets when the softest jet is in a possible extension of the CMS CASTOR detector, while the hard jet is around CMS HF detector.

\section{Acknowledgments}

The author thanks for the support from the NCBiR grant LIDER/02/35/L-2/10/NCBiR/2011.

\section{References}

[1] V. S. Fadin, E. Kuraev, and L. Lipatov, On the Pomeranchuk Singularity in Asymptotically Free Theories, Phys.Lett. B60 (1975) 50-52

[2] E. Kuraev, L. Lipatov, and V. S. Fadin, The Pomeranchuk Singularity in Nonabelian Gauge Theories, Sov.Phys.JETP 45 (1977) 199-204

[3] I. Balitsky and L. Lipatov, The Pomeranchuk Singularity in Quantum Chromodynamics, Sov.J.Nucl.Phys. 28 (1978) 822-829

[4] I. Balitsky, Operator expansion for high-energy scattering, Nucl.Phys. B463 (1996) 99-160 [arXiv:hep-ph/9509348]

[5] Y. V. Kovchegov, Small x $F(2)$ structure function of a nucleus including multiple pomeron exchanges, Phys.Rev. D60 (1999) 034008 [arXiv: hep-ph/9901281]

[6] I. Balitsky and G. A. Chirilli, Next-to-leading order evolution of color dipoles, Phys.Rev. D77 (2008) 014019 [arXiv:0710.4330]

[7] J. L. Albacete, N. Armesto, J. G. Milhano, and C. A. Salgado, Non-linear QCD meets data: A Global analysis of lepton-proton scattering with running coupling BK evolution, Phys.Rev. D80 (2009) 034031 [arXiv:0902.1112]

[8] L. Gribov, E. Levin, and M. Ryskin, Semihard Processes in QCD, Phys.Rept. 100 (1983) 1-150

[9] M. Ciafaloni, Coherence Effects in Initial Jets at Small $q^{* * 2}$ / s, Nucl.Phys. B296 (1988) 49

[10] S. Catani, F. Fiorani, and G. Marchesini, Small x Behavior of Initial State Radiation in Perturbative QCD, Nucl.Phys. B336 (1990) 18

[11] S. Catani, F. Fiorani, and G. Marchesini, QCD Coherence in Initial State Radiation, Phys.Lett. B234 (1990) 339

[12] K. Kutak, K. Golec-Biernat, S. Jadach, and M. Skrzypek, Nonlinear equation for coherent gluon emission, JHEP 1202 (2012) 117 [arXiv:1111.6928]

[13] K. Kutak and J. Kwiecinski, Screening effects in the ultrahigh-energy neutrino interactions, Eur.Phys.J. C29 (2003) 521 [arXiv: hep-ph/0303209] 
[14] K. Kutak and A. Stasto, Unintegrated gluon distribution from modified BK equation, Eur.Phys.J. C41 (2005) 343-351 [arXiv: hep-ph/0408117]

[15] K. Kutak and S. Sapeta, Gluon saturation in dijet production in p-Pb collisions at Large Hadron Collider, Phys.Rev. D86 (2012) 094043 [arXiv: 1205.5035$]$

[16] S. Catani, M. Ciafaloni, and F. Hautmann, High-energy factorization and small $x$ heavy flavor production, Nucl.Phys. B366 (1991) 135-188

[17] J. C. Collins and R. K. Ellis, Heavy quark production in very high-energy hadron collisions, Nucl. Phys. B360 (1991) 3-30

[18] E. Avsar, On the Understanding and Use of 'Unintegrated' Parton Distributions in Small-x QCD, Int.J.Mod.Phys.Conf.Ser. 04 (2011) 74-84 [arXiv : 1108.1181$]$

[19] E. Avsar, TMD factorization and the gluon distribution in high energy QCD, arXiv:1203.1916

[20] P. Mulders and T. Rogers, Gauge Links, TMD-Factorization, and TMD-Factorization Breaking, arXiv: 1102.4569

[21] B.-W. Xiao and F. Yuan, Non-Universality of Transverse Momentum Dependent Parton Distributions at Small-x, Phys.Rev.Lett. 105 (2010) 062001 [arXiv: 1003.0482 ]

[22] F. Dominguez, C. Marquet, B.-W. Xiao, and F. Yuan, Universality of Unintegrated Gluon Distributions at small x, Phys.Rev. D83 (2011) 105005 [arXiv: 1101.0715$]$

[23] M. Deak, F. Hautmann, H. Jung, and K. Kutak, Forward Jet Production at the Large Hadron Collider, JHEP 0909 (2009) 121 [arXiv: 0908 . 0538]

[24] E. Antonov, L. Lipatov, E. Kuraev, and I. Cherednikov, Feynman rules for effective Regge action, Nucl.Phys. B721 (2005) 111-135 [arXiv: hep-ph/0411185]

[25] A. van Hameren, P. Kotko, and K. Kutak, Multi-gluon helicity amplitudes with one off-shell leg within high energy factorization, JHEP 1212 (2012) 029 [arXiv: 1207.3332]

[26] A. van Hameren, P. Kotko, and K. Kutak, Helicity amplitudes for high-energy scattering, JHEP 1301 (2013) 078 [arXiv:1211.0961]

[27] A. van Hameren, BCFW recursion for off-shell gluons, arXiv:1404.7818

[28] P. Kotko, Wilson lines and gauge invariant off-shell amplitudes, arXiv: 1403.4824

[29] P. Kotko, OGIME - Off-shell Gauge Invariant Matrix Elements, 2013, FORM program, http: //annapurna.ifj.edu.pl/ pkotko/OGIME.html

[30] A. van Hameren, P. Kotko, K. Kutak, C. Marquet, and S. Sapeta, Saturation effects in forward-forward dijet production in p+Pb collisions, Phys.Rev. D89 (2014) 094014 [arXiv: 1402 . 5065]

[31] S. Sapeta, forward, the code is available on request from the author

[32] P. Kotko, LxJet, 2013, C++ Monte Carlo program, http://annapurna.ifj.edu.pl/ pkotko/LxJet.html

[33] A. van Hameren, P. Kotko, and K. Kutak, Three jet production and gluon saturation effects in p-p and p-Pb collisions within high-energy factorization, arXiv: 1308.0452

[34] A. van Hameren, P. Kotko, K. Kutak, and S. Sapeta, Small-x dynamics in forward-central dijet decorrelations at the LHC, arXiv: 1404.6204

[35] CMS Collaboration, Measurement of azimuthal correlations between forward and central jets in proton proton collisions at $\operatorname{sqrt}(\mathrm{s})=7 \mathrm{TeV}$, 